\title{
Robot-Assisted Surgery and Holmium Laser in Complex Choledocholithiasis
}

\author{
Alexis Sanchez, MD, MSc, Omaira Rodriguez, MD, Renata Sanchez, MD, Luis Medina, MD, \\ Liumariel Vegas, MD, Génesis Jara, MD, José Rosciano, MD \\ Robotic Surgery Program, University Hospital of Caracas, Caracas, Venezuela (all authors).
}

\begin{abstract}
Introduction: Unsolved choledocholithiasis by endoscopic retrograde cholangiopancreatography is a complicated condition to manage. The incorporation of robotic surgery, a choledochoscope, and a holmium laser as therapeutic tools is likely to increase surgery effectiveness. Our purpose is to present the first Latin American case report of the combined use of these tools to extract a large impacted bile duct stone.

Case Description: A 42-year-old man with obstructive jaundice syndrome underwent endoscopic retrograde cholangiopancreatography. Bile duct dilatation was evidenced by a 1.5 -cm-diameter stone that could not be extracted, and biliary prosthesis placement was not possible. Given that stone clearance was not achieved, robot-assisted laparoscopic common bile duct exploration using a holmium laser for lithotripsy was performed. The entire procedure was performed with a robot-assisted technique. The docking time and console time were 10 minutes and 120 minutes, respectively. The patient progressed satisfactorily and was discharged after 48 hours without complications. He had no evidence of residual common bile duct stones or duct strictures after 12 months of follow-up.
\end{abstract}

Discussion: Laparoscopic common bile duct exploration is an excellent option for the treatment of common bile duct stones. The holmium laser is a useful tool for the treatment of complex choledocholithiasis. Incorporation of the da Vinci System (Intuitive Surgical, Sunnyvale, California) allows greater precision and effectiveness, thus increasing surgery success rates.

Key Words: Robotic surgery, Choledocholithiasis, Holmium laser.

Citation Sanchez A, Rodriguez O, Sanchez R, Medina L, Vegas L, Jara G, Rosciano J. Robot-Assisted surgery and holmium laser in complex choledocholithiasis. CRSLS e2015.00014. DOI: $10.4293 /$ CRSLS.2015.00014

Copyright $(0) 2015$ by SLS, Society of Laparoendoscopic Surgeons. This is an open-access article distributed under the terms of the Creative Commons Attribution-Noncommercial-ShareAlike 3.0 Unported license, which permits unrestricted noncommercial use, distribution, and reproduction in any medium, provided the original author and source are credited.

A.S. is proctor of robotic surgery. The other authors have no conflict of interest or financial ties to disclose.

Address correspondence to: Alexis Sanchez, MD, MSc, Robotic and Minimally Invasive Surgery Center, La Floresta Medical Institute, Av. Principal La Floresta, Caracas, Miranda 1060, Venezuela. Telephone: 58414 3310944, E-mail: dralexissanchez@hotmail.com

\section{INTRODUCTION}

The presence of stones in the bile duct is considered a challenge in its resolution. Currently, the most accepted treatment options include a 2-step resolution by endoscopic retrograde cholangiopancreatography (ERCP) with preoperative endoscopic sphincterotomy, followed by laparoscopic cholecystectomy. When ERCP is inefficient because of intrahepatic or large impacted stones in the bile duct, the next step is surgical exploration of the biliary tract. ${ }^{1}$

We have shown the effectiveness of laparoscopy in the management of choledocholithiasis. In patients in whom
ERCP is ineffective, the laparoscopic conversion rate increases mainly because of impacted stones that require complex management. ${ }^{2}$

The incorporation of new technologies such as robotic surgery and a holmium laser in the management of choledocholithiasis increases the success rate, allowing proper handling of complex cases. ${ }^{3,4}$ Robot-assisted surgery helps to overcome difficulties of other minimally invasive approaches, providing a 3-dimensional view and high maneuverability of the instruments. The holmium laser allows fragmentation of large impacted stones and 


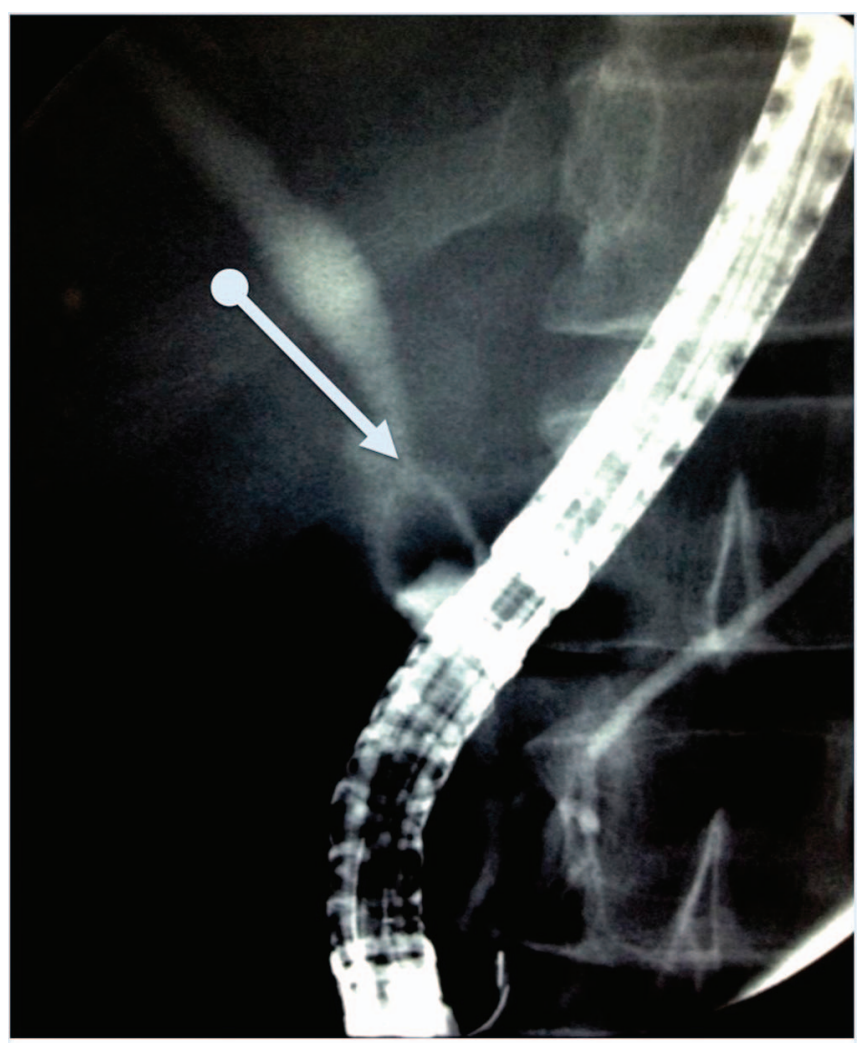

Figure 1. Endoscopic retrograde cholangiopancreatography. The arrow indicates a large impacted stone in the common bile duct.

their subsequent removal using conventional methods such as balloons or helicoid baskets.

The main purpose of this article is to present the first Latin American experience in the combined use of these new technologies-robotic surgery, choledochoscopy, and holmium laser-in the management of complex choledocholithiasis.

\section{CASE DESCRIPTION}

A 42-year-old male patient underwent a consultation regarding obstructive jaundice syndrome. Physical examination findings at admission showed the following: normal vital signs, a soft and depressible abdomen that was painful to deep palpation in the epigastrium and right upper quadrant, no signs of peritoneal irritation, the presence of bowel sounds, and no evidence of organ enlargement. Paraclinical studies were conducted and showed an elevated bilirubin level, an alkaline phosphatase level of $320 \mathrm{U} / \mathrm{mL}$, and a $\gamma$-glutamyl transpeptidase level of 440
$\mathrm{U} / \mathrm{mL}$. Abdominal ultrasonography was performed and showed gallstones and a bile duct diameter of $20 \mathrm{~mm}$.

The patient was admitted with a diagnosis of gallstones and high risk of choledocholithiasis. According to our department protocol for patients with suspected choledocholithiasis, ERCP was conducted. 5 Bile duct dilatation was evidenced with a $1.5-\mathrm{cm}$-diameter stone that could not be extracted, and biliary prosthesis placement was not possible (Figure 1). The patient was referred to undergo surgical management, and robot-assisted laparoscopic common bile duct exploration (LCBDE) using a holmium laser for lithotripsy was scheduled.

\section{SURGICAL TECHNIQUE}

LCBDE was performed according to the technique described by Sánchez et al. ${ }^{4}$ The patient was placed in the supine position. Five operating ports were used: An infraumbilical 12-mm camera port was placed first, followed by placement of two 8-mm ports for arms 1 and 2, as shown in Figure 2. We also used a $12-\mathrm{mm}$ port for the choledochoscope and a $5-\mathrm{mm}$ port for retraction.

Robotic system docking was performed over the right shoulder of the patient. Then, after the Calot triangle was identified and the critical view was obtained, a 7-mm-long supraduodenal choledochotomy was performed (Figure 3). A transcystic approach was not intended because of the choledochoscope's diameter of $4.9 \mathrm{~mm}$ and nondilated cystic duct.

The choledochoscope was introduced, and a proximal inspection was performed, showing no evidence of secondary biliary tract stones. On distal inspection, we iden-

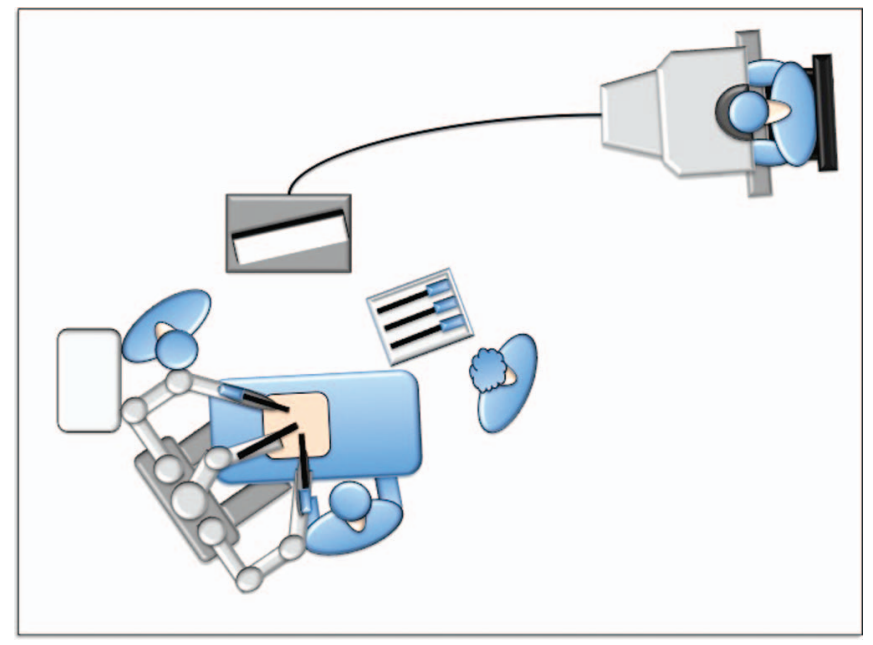

Figure 2. Operating room and trocar positioning. 


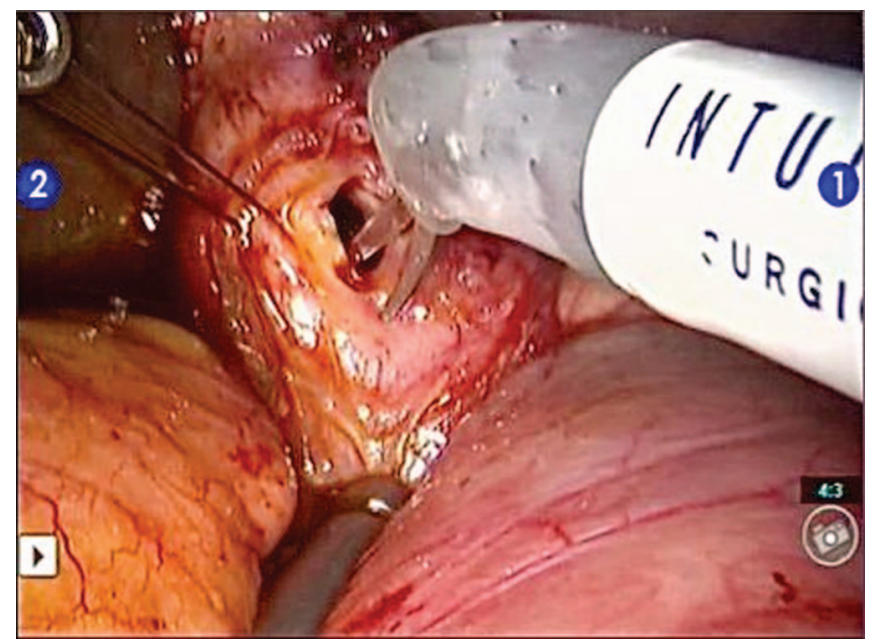

Figure 3. Longitudinal supraduodenal choledochotomy using EndoWrist instruments.

tified a $1.5-\mathrm{cm}$ stone; extraction with a Dormia basket and Fogarty balloons was attempted but yielded no results. Therefore, we used a 200- $\mu \mathrm{m}$ holmium laser fiber (pulse, $0.6 \mathrm{~J}$ at $6 \mathrm{~Hz}$ ) through the choledochoscope's working channel, achieving stone fragmentation (Figure 4). The stone's fragments were removed using a helicoid basket and continuous irrigation with saline solution.

Final inspection was performed, and no residual stones in the biliary tract were found (Figure 5). Finally, we performed choledochotomy primary closure using separate stitches with a resorbable No. 4-0 suture.

The procedure was finalized with standard cholecystectomy and subhepatic drain placement. The docking time was 10 minutes, and the console time was 120 minutes. The length of stay was 48 hours. At a follow-up time of 12 months, no duct-related complications have been reported.

\section{DISCUSSION}

Laparoscopic common bile duct exploration has proved to be a procedure that provides high success rates and low
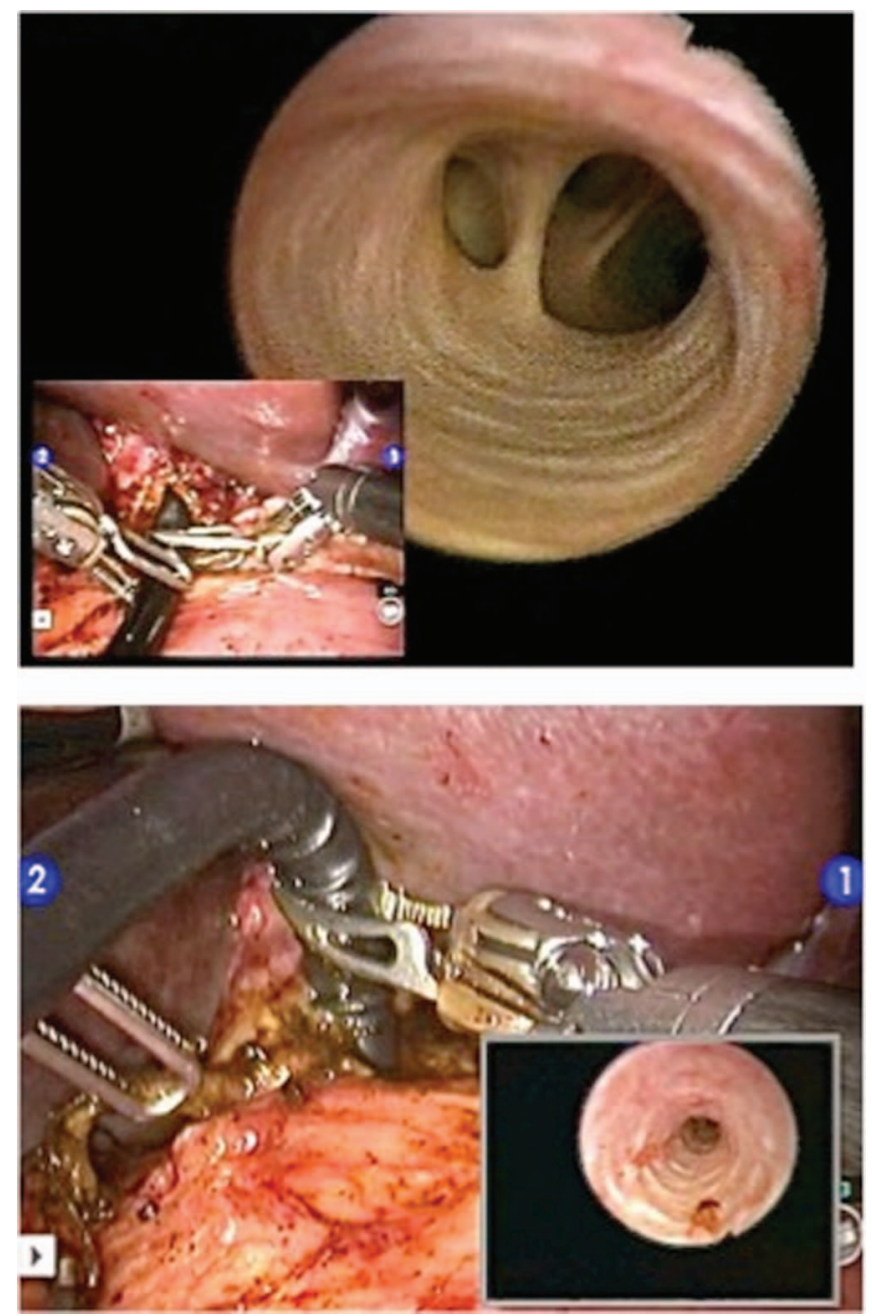

Figure 5. Final revision. Proximal and distal view using choledochoscope.

morbidity rates in the treatment of gallstones complicated by choledocholithiasis. After 9 years of experience, we have achieved an $84 \%$ efficiency rate in managing these conditions. 6,7
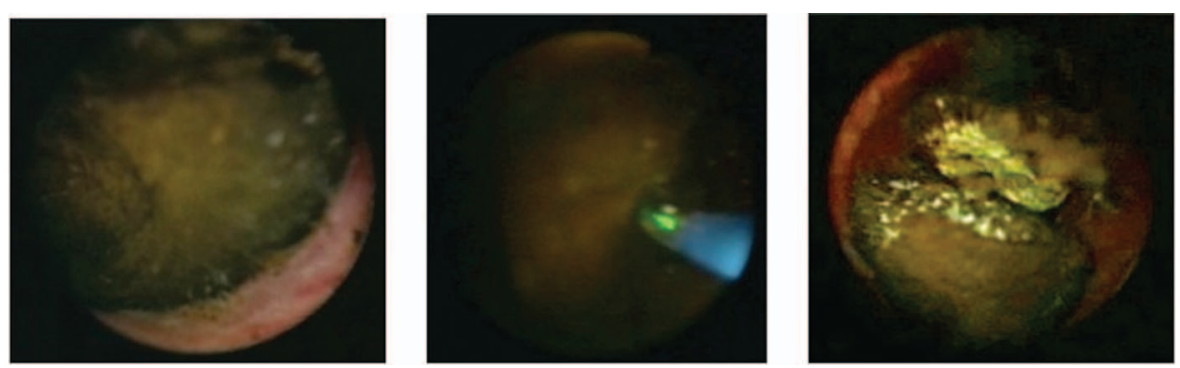

Figure 4. Impacted stone fragmentation using holmium laser through choledochoscope working channel. 
The advantages of the robotic surgery platform have a significant impact on the ability of surgeons to perform complex procedures. Robot-assisted surgery improves laparoscopy procedures, providing a 3-dimensional view, increasing the degrees of movement, eliminating the fulcrum effect, and optimizing ergonomics, which reduces physical and mental stress. This technology also offers other advantages, such as eliminating tremor in the surgeon's hands and providing a stable picture and camera control. Roeyen et al, ${ }^{8}$ in 2004 , reported the first robotassisted common bile duct exploration in the literature; we published our initial experience in 2010.4 To date, there have been few reports and short series regarding this procedure. $3,4,9$

The EndoWrist instrument (Intuitive Surgical, Sunnyvale, California) enhances the dexterity of the surgeon, improving precision and control beyond the human hand's capabilities, in addition to allowing a better dissection of the biliary tract, as well as choledochotomy, and an optimal manipulation of the choledochoscope, which facilitates the exploration. ${ }^{9,10}$ Robotic surgery appears to decrease the surgical time even if we add the docking phase of the robot. In our case the docking time was 10 minutes, with a console time of 120 minutes, similar to our conventional laparoscopic procedures' mean time.

The surgical robot offers the potential to integrate multiple views into the surgical console screen to provide real-time views of both fields of operation, which in this case means visualization of the laparoscopic optic and the choledochoscope simultaneously. This function (da Vinci Surgical System's Tilepro multi-display system; Intuitive Surgical) has the potential to increase patient safety and surgical efficiency during procedures. Surgeons are able to use the technologies incorporated into the robot system to maximize patient benefit. ${ }^{11}$ As an additional advantage, the robotic arms and EndoWrist would reach and capture common bile duct stones with greater ease.

The main causes of failure in LCBDE usually are intrahepatic and impacted bile duct stones. In these cases the use of methods to achieve stone fragmentation increases the effectiveness of LCBDE. ${ }^{1,2}$

The first experience using laser lithotripsy for the treatment of bile duct stones is attributed to Orii et al ${ }^{12}$ in 1983, who reported 8 patients with intrahepatic stones and 3 patients with choledocholithiasis treated with an yttriumaluminum-garnet laser. The procedure was performed shortly after the ineffectiveness of the endoscopic treatment was shown.
The use of the holmium laser in the treatment of common bile duct stones has been reported in several studies. Shamamian and Grasso, ${ }^{13}$ in 2004, conducted a study of 36 patients with a diagnosis of complex choledocholithiasis who underwent lithotripsy with the holmium laser using a flexible choledochoscope; they concluded that fragmentation and stone removal could be achieved safely with the laser.

The holmium laser is composed of a mineral compound called holmium, which gives amplified energy in pulses, achieving photoacoustic ablation of stones regardless of their composition, size, or location without damaging underlying structures. ${ }^{14}$ The laser uses a fiberoptic system; the fiber delivers energy between 0.6 and $1 \mathrm{~J}$ with frequencies ranging between 6 and $10 \mathrm{~Hz}$. The depth of penetration is limited to 0.5 to $1 \mathrm{~mm}$, and the photoacoustic effect produces minimal retropulsion and reduces the risk of injury due to mechanical force.

The use of the holmium laser and choledochoscope requires a great deal of coordination between the console surgeon and the surgeon operating the endoscope. We have previously reported the usefulness of simulation with inanimate models to achieve optimal planning and coordination outside the operating room..$^{4,15}$

The role of robotic surgery in the treatment of choledocholithiasis is still to be decided. There are few reports for the time being. This is the first Latin American case report that includes both the holmium laser and robotic surgery in the laparoscopic management of common bile duct stones. The incorporation of these technologies will increase surgery success rates, thus providing minimally invasive surgery benefits to a greater number of patients.

\section{References:}

1. Kharbutli B, Velanovich V. Management of preoperatively suspected choledocholithiasis: a decision analysis. J Gastrointest Surg. 2008;12:1973-1980.

2. Sanchez A, Rodriguez O, Bellorín O, Sánchez R, Benítez G. Laparoscopic common bile duct exploration in patients with gallstones and choledocholithiasis. JSLS. 2010;14:246-250.

3. Alkhamesi NA, Davies WT, Pinto RF, Schlachta CM. Robotassisted common bile duct exploration as an option for complex choledocholithiasis. Surg Endosc. 2013;27:263-266.

4. Sánchez A, Rodríguez O, Davila H, et al. Robot-assisted laparoscopic common bile duct exploration: case report and proposed training model. J Robotic Surg. 2011;5(2):145-148. 
5. Sánchez-Ismayel A, Rodríguez O, Sánchez R. Colangiografía intraoperatoria selectiva y manejo laparoscópico en un solo tiempo de la colédocolitiasis. Rev Venez Cir. 2008;61(4):155-161.

6. Sánchez-Ismayel A, Rodríguez O, Benítez G, Sánchez R, Bellorín O. Exploración laparoscópica de la vía biliar y manejo actual de la coledocolitiasis. Análisis de los primeros 30 casos. Rev Venez Cir. 2010;63:65-71.

7. Sánchez-Ismayel A, Rodríguez O, Sánchez R, Benítez G, Raga L, Jara G. Cierre primario de la vía biliar versus cierre sobre tubo en "T" durante exploración laparoscópica de la vía biliar. Rev Venez Cir. 2013;66:93-97.

8. Roeyen G, Chapelle T, Ysebart D. Robot-assisted choledochotomy. Surg Endosc. 2004;18:165-166.

9. Ji WB, Zhao ZM, Dong JH, Wang HG, Lu F, Lu HW. Onestage robotic-assisted laparoscopic cholecystectomy and common bile duct exploration with primary closure in 5 patients. Surg Laparosc Endosc Percutan Tech. 2011;21(2):123-126.

10. Jayaraman S, Davies W, Schlachta C. Robot-assisted minimally invasive common bile duct exploration: a Canadian first. Can J Surg. 2008;51(4):93-94.
11. Woo Y, Choi GH, Min BS, Hyung WJ. Novel application of simultaneous multi-image display during complex robotic abdominal procedures. BMC Surg. 2014;14:13.

12. Orii K, Ozaki A, Takase Y, Iwasaki Y. Lithotomy of intrahepatic and choledochal stones with Yag laser. Surg Gynecol Obstet. 1983;4:485-488.

13. Shamamian P, Grasso M. Management of complex biliary tract calculi with a holmium laser. J Gastrointest Surg. 2004;8: 191-199.

14. Varban O, Assimos D, Passman C, Westcott C. Laparoscopic common bile duct exploration and holmium laser lithotripsy: a novel approach to the management of common bile duct stones. Surg Endosc. 2010;24:1759-1764.

15. Sanchez A, Otaño N, Rodríguez O, Sanchez R, Benítez G, Schweitzer M. Laparoscopic common bile duct exploration fourtask training model: construct validity. JSLS. 2012;16:10-15. 